\title{
CCAAT Enhancer Binding Protein
}

National Cancer Institute

\section{Source}

National Cancer Institute. CCAAT Enhancer Binding Protein. NCI Thesaurus. Code C17486.

A protein which binds to enhancers having a conserved CCAAT sequence. CCAAT enhancer binding proteins are a subclass of bZIP transcription factors that bind the CCAAT DNA sequence as homo- or heterodimers with other family members, C/EBPalpha, beta, gamma, delta, epsilon. They include both transcriptional activators and repressors, and some are involved in myelopoiesis. 